\title{
ON STABILITY OF ENDOMORPHISMS
}

\author{
HIROSHI IKEDA
}

(Communicated by George R. Sell)

\begin{abstract}
In this note we prove a generalization of R. Mañe's theorem. $R$. Mañe proved that $C^{r}$ absolutely stable endomorphisms satisfy Axiom A. We prove that if an endomorphism $f$ is both $C^{r}$ structurally and infinitesimally stable, then $f$ satisfies Axiom A.
\end{abstract}

1. Introduction. This note is a generalization of R. Mañé's theorem on absolute stability [4]. We prove this theorem under weaker hypotheses. Our Theorem is the following

THEOREM. If $f \in \operatorname{End}^{r}(M)$ is both $C^{r}$ structurally and infinitesimally stable, then $f$ satisfies Axiom A.

First we establish some background. Let $M$ be a compact connected smooth manifold without boundary and let $\operatorname{End}^{r}(M), r \geq 1$, be the space of $C^{r}$ endomorphisms of $M$ endowed with the $C^{r}$ topology. We say that $f$ is $C^{r}$ structurally stable if there exists a neighborhood $\mathscr{U}$ of $f$ in $\operatorname{End}^{r}(M)$ such that for every $g \in \mathscr{U}$ there exists a homeomorphism $h$ of $M$ satisfying $f h=h g$. If $f \in \operatorname{End}^{r}(M)$, let $\operatorname{Per}(f)$ be the set of all periodic points of $f$ and let $\Omega(f)=\{x \in M \mid$ for every neighborhood $U$ of $x$, there exists $n>0$ with $\left.f^{n}(U) \cap U \neq \varnothing\right\}$. We denote by $S(f)$ the set of singularities of $f$, i.e. those points $x$ of $M$ where $T f \mid T_{x} M$ is not injective.

We give two definitions following $\mathrm{R}$. Mañé [4].

DEFINITION. We say that $f \in \operatorname{End}^{r}(M)$ is $C^{r}$ absolutely stable if there exist a neighborhood $\mathscr{U}$ of $f$ in $\operatorname{End}^{r}(M)$ and a constant $K>0$ such that for all $g \in \mathscr{U}$ there exists a homeomorphism $h$ of $M$ satisfying $g h=h f$ and $d(h, I) \leq K d(f, g)$ where $d(\cdot, \cdot)$ is defined by $d\left(f_{1}, f_{2}\right)=\sup \left\{\rho\left(f_{1}(x), f_{2}(x)\right) \mid x \in M\right\}, \rho(\cdot, \cdot)$ being a metric on $M$ and $I$ is the identity map of $M$.

Definition. We say that $f \in \operatorname{End}^{r}(M)$ satisfies Axiom A if there exist a continuous splitting $T M \mid \Omega(f)=E^{s} \oplus E^{u}$, and a Riemannian metric | | on $M$, and constants $K>0,0<\lambda<1$ satisfying:

(a) $(T f) E^{s} \subset E^{s},(T f) E^{u}=E^{u}$;

$$
\begin{aligned}
& \left|(T f)^{n}\right| E_{x}^{s} \mid \leq K \lambda^{n} \quad \text { for } x \in \Omega(f), n>0, \\
& \left|(T f)^{n} v\right| \geq K \lambda^{-n}|v| \quad \text { for } x \in \Omega(f), v \in E_{x}^{u}, n>0 ;
\end{aligned}
$$

(c) if $x_{1} \neq x_{2} \in \Omega(f)$ and $f\left(x_{1}\right)=f\left(x_{2}\right)=x$, then $E_{x}^{s}=\{0\}$;

(d) $\operatorname{Per}(f)$ is dense in $\Omega(f)$;

(e) $S(f) \cap \Omega(f)=\varnothing$.

In [8] the following conjecture was stated.

Received by the editors September 29, 1986.

1980 Mathematics Subject Classification (1985 Revision). Primary 58F 10; Secondary 58F15.

Key words and phrases. Infinitesimal stability, Axiom A, prehyperbolic set, $\bar{P}$-stability. 
Conjecture. If $f \in \operatorname{End}^{r}(M)$ is $C^{r}$ structurally stable, then $\Omega(f) \cap S(f)=\varnothing$. In fact, $\operatorname{Per}(f) \cap S(f)=\varnothing$ holds if $f$ is $C^{r}$ structurally stable, and $\Omega(f) \cap S(f)=$ $\varnothing$ holds if $f$ is $C^{r}$ absolutely stable. The latter property is a part of the theorem by R. Mañé [4].

THEOREM M. $C^{r}$ absolutely stable endomorphisms satisfy Axiom A.

On the other hand, F. Przytycki defined Axiom A somewhat different from Mañé's Axiom A, and found sufficient conditions for $\Omega$-stability of an endomorphism $[6,7]$.

Our Theorem is a generalization of Theorem $\mathrm{M}$.

2. Proof of the Theorem. In this section we will prove our Theorem. To prove the Theorem we need some definitions and lemmas. If $\Lambda \subset M$ is a compact subset of $M$ let $\Gamma^{b}(\Lambda)$ be the space of bounded sections of $T M \mid \Lambda$ with the norm $\|\eta\|=\sup \{\|\eta(x)\| \mid x \in \Lambda\}$ and let $\Gamma^{0}(\Lambda)$ be the closed subspace of continuous sections. If $f \in \operatorname{End}^{r}(M)$ and $f(\Lambda) \subset \Lambda$ let $T_{f} M \mid \Lambda$ be the vector bundle on $\Lambda$ consisting of couples $(p, v)$ with $p \in \Lambda, v \in T_{f(p)} M$. Let $\Gamma_{f}^{b}(\Lambda), \Gamma_{f}^{0}(\Lambda)$ be the corresponding spaces of bounded and continuous sections of $T_{f} M \mid \Lambda$.

We define the linear operator $L_{f}: \Gamma^{b}(\Lambda) \rightarrow \Gamma_{f}^{b}(\Lambda)$ by

$$
L_{f}(\eta)=(T f) \circ \eta-\eta \circ f \text { for } \eta \in \Gamma^{b}(\Lambda) .
$$

DEFinition. Let $f \in \operatorname{End}^{r}(M)$ and let $\Lambda \subset M$ be a compact subset with $f(\Lambda)=\Lambda$. We say that $\Lambda$ is a prehyperbolic set for $f$ if there exist a continuous splitting $T M \mid \Lambda=E^{s} \oplus E^{u}$, and a Riemannian metric || on $M$ and constants $K>0$, $0<\lambda<1$ satisfying:

(a) $(T f) E^{s} \subset E^{s},(T f) E^{u}=E^{u}$;

$$
\begin{aligned}
& \left|(T f)^{n}\right| E_{x}^{s} \mid \leq K \lambda^{n} \text { for } x \in \Lambda, n>0 \\
& \left|(T f)^{n} v\right| \geq K \lambda^{-n}|v| \text { for } x \in \Lambda, v \in E_{x}^{u}, n>0
\end{aligned}
$$

(c) if $x_{1} \neq x_{2} \in \Lambda$ and $f\left(x_{1}\right)=f\left(x_{2}\right)=x$, then $E_{x}^{s}=\{0\}$.

We say that $f \in \operatorname{End}^{r}(M)$ is infinitesimally stable if the linear operator $L_{f}$ : $\Gamma^{0}(M) \rightarrow \Gamma_{f}^{0}(M)$ is surjective. It is evident that the absolute stability of $f$ implies the structural stability of $f$. Also it is easy to prove that if $f \in \operatorname{End}^{r}(M)$ is absolutely stable, then $f$ is infinitesimally stable by the similar argument for the case of diffeomorphisms in [1]. If $x \in M$ let $\omega(x)=\omega(x, f)$ be the set of $\omega$-limit points of $x$ for $f$ and let $\bar{L}^{+}(f)$ be the closure of $L^{+}(f)=\{\omega(x) \mid x \in M\}$.

In the proof of the theorem, we shall use the following two lemmas of [4].

LEMMA 1. If $f \in \operatorname{End}^{r}(M)$ is infinitesimally stable, then $\bar{L}^{+}(f)$ is prehyperbolic.

LEMMA 2. Suppose that $f \in \operatorname{End}^{r}(M)$ and $\bar{L}^{+}(f)$ is prehyperbolic. Then given $x \in \bar{L}^{+}(f)$ and a neighborhood $\mathscr{U}$ of $f$ there exist a neighborhood $U$ of $x$ and $g \in \mathscr{U}$ such that:

(a) $g(y)=f(y)$ for all $y \in U$;

(b) $x \in \operatorname{Per}(g)$.

From the argument of R. Mañé [4], it clearly follows that we only need to prove the following proposition in order to prove the Theorem. 
PROPOSITION. If $f \in \operatorname{End}^{r}(M)$ is both $C^{r}$ structurally and infinitesimally stable, then $\bar{L}^{+}(f)=\overline{\operatorname{Per}(f)}$.

To prove the Proposition, we introduce the concept of $\bar{P}$-stability. $\bar{P}=\overline{\operatorname{Per}(f)}$ will denote the closure of the set of periodic points of $f$. We say that $f \in \operatorname{End}^{r}(M)$ is $\bar{P}$-stable if there exists a neighborhood $\mathscr{U}$ of $f$ in $\operatorname{End}^{r}(M)$ such that for each $g \in \mathscr{U}$ there exists a homeomorphism $h: \overline{\operatorname{Per}(f)} \rightarrow \overline{\operatorname{Per}(g)}$ satisfying $h f=g h$ [5]. It is obvious that the structural stability implies the $\bar{P}$-stability.

Proof of Proposition. We first suppose that $\overline{\operatorname{Per}(f)} \subsetneq \bar{L}^{+}(f)$. We show that $\bar{L}^{+}(f) \cap S(f)=\varnothing$. Suppose that $x \in \bar{L}^{+}(f) \cap S(f)$. By Lemmas 1 and 2, we can take $g \in \operatorname{End}^{r}(M)$ nearby $f$ coinciding with $f$ in a neighborhood of $x$ (therefore $x \in S(g)$ ) and such that $x \in \operatorname{Per}(g)$. Hence $x \in \operatorname{Per}(g) \cap S(g)$. This contradicts the stability of $g$. Hence $\bar{L}^{+}(f) \cap S(f)=\varnothing$. Also it is obvious that $\overline{\operatorname{Per}(f)}$ is prehyperbolic. Therefore $\overline{\operatorname{Per}(f)}$ has a decomposition, $\Lambda_{1} \cup \Lambda_{2} \cup \cdots \cup \Lambda_{n}$ into disjoint prehyperbolic sets, following the method of Przytycki [7] and Newhouse [5]. Here each $\Lambda_{i}$ is a prehyperbolic set such that the periodic points are dense in $\Lambda_{i}$. Let $U_{i}$ be a compact neighborhood of $\Lambda_{i}$ for $1 \leq i \leq n$. If we choose $U_{i}$ small enough, we have that $U_{i} \cap U_{j}=\varnothing$ if $i \neq j$ and $\bar{L}^{+}(f) \backslash \bigcup_{1<i<n} U_{i} \neq \varnothing$.

Now suppose that $f \mid \Lambda_{i}$ is injective. Then shrinking $U_{i}$ if necessary, $f \mid U_{i}$ is a diffeomorphism onto its image. By Theorem 7.3 of [2], there is a neighborhood $\mathscr{N}_{i}$ of $f$ in $\operatorname{End}^{r}(M)$ such that if $g \in \mathscr{N}_{i}$ then there exists a homeomorphism $h$ close to identity which conjugates $\Lambda_{i}$ with a $g$-invariant subset of $U_{i}$.

Next suppose that $f \mid \Lambda_{i}$ is not injective. Then it is easy to show that $f \mid \Lambda_{i}$ is a (quasi-) expanding map, i.e. $\operatorname{dim} E_{x}^{u}=\operatorname{dim} M$ for any $x \in \Lambda_{i}$. By the similar argument to Przytycki [7], there exists a neighborhood $\mathscr{N}_{i}$ of $f$ in $\operatorname{End}^{r}(M)$ such that if $g \in \mathscr{N}_{i}$ then there exists a homeomorphism $h$ from $\Lambda_{i}$ onto its image satisfying $g h=h f$ and $h\left(\Lambda_{i}\right) \subset U_{i}$.

From above arguments and $\bar{P}$-stability of $f$, it follows that there exists a neighborhood $\mathscr{N}$ of $f$ in $\operatorname{End}^{r}(M)$ such that $\overline{\operatorname{Per}(g)} \subset \bigcup_{1 \leq i \leq n} U_{i}$ for any $g \in \mathscr{N}$. Let $x \in \bar{L}^{+}(f) \backslash \bigcup_{1 \leq i \leq n} U_{i}$. By Lemma 2 , there exists $g \in \mathscr{N}$ such that $x$ is a periodic point of $g$. This is a contradiction.

Subsequently we obtain the Corollary of the Theorem:

COROLLARY. If $f \in \operatorname{End}^{r}(M)$ is both $C^{r}$ structurally and infinitesimally stable, then $f$ satisfies Axiom A and no cycle condition.

\section{REFERENCES}

1. J. Guckenheimer, Absolutely $\Omega$-stable diffeomorphisms, Topology 11 (1972), 195-197.

2. M. Hirsch and C. Pugh, Stable manifolds and hyperbolic sets, Proc. Sympos. Pure Math., vol. 14, Global Analysis, Amer. Math. Soc., Providence, R.I., 1970, pp. 133-163.

3. R. Mañé, On infinitesimal and absolute stability of diffeomorphisms, Dynamical Systems (Warwick, 1974), Lecture Notes in Math., vol. 468, Springer-Verlag, Berlin, Heidelberg and New York, pp. 151-161.

4. __ Axiom A for endomorphisms, Lecture Notes in Math., vol. 597, Springer-Verlag, Berlin, Heidelberg and New York, pp. 379-388.

5. S. Newhouse, Hyperbolic limit sets, Trans. Amer. Math. Soc. 67 (1972), 125-150.

6. F. Przytycki, Anosov endomorphisms, Studia Math. 58 (1976), 249-285. 
7. _ On $\Omega$-stability and structural stability of endomorphisms satisfying Axiom A, Studia Math. 60 (1977), 61-77.

8. C. Pugh and R. Mañé, Stability of endomorphisms, Lecture Notes in Math., vol. 468, SpringerVerlag, Berlin, Heidelberg and New York, 1975, pp. 175-184.

Department of Mathematics, SChool of Science and Engineering, Waseda UNIVERSITY, SHINJUKU, TOKYO, JAPAN 